\title{
AN ESTIMATE OF THE PERCOLATION PARAMETER IN HEAVY ION COLLISIONS
}

\author{
J.Dias de Deus and Yu. M. Shabelski* \\ CENTRA, Instituto Superior Técnico, 1049-001 Lisboa, Portugal
}

\begin{abstract}
From existing hadron and heavy ion collisions data on $\bar{p} / p$ central production we estimate the value of the percolation parameter at RHIC energies.

*Permanent address: Petersburg Nuclear Physics Institute, Gatchina, St.Petersburg, Russia

E-mail: shabelsk@thd.pnpi.spb.ru
\end{abstract}


It is well-known that in high energy hadron-nucleus collision there exists inelastic screening [1, 2] experimentally confirmed, especially for the case of hadron-deuteron interactions. The same inelastic screening has to exist in high energy heavy ion collision, as well. This effect is very small for integrated cross sections (because many of them are determined by geometry), but it is very important [3] for the calculations of secondary multiplicities and inclusive densities. Similar results are obtained $[4,5]$ in the framework of string fusion [6], or percolation [7] models, where string fusion/percolation effects directly correspond [8] to pomeron interactions and are responsible for the suppression of particle production.

The effect of percolation for central heavy ion collision is, in good approximation determined by the reduction factor [9]

$$
F(\eta)=\sqrt{\frac{1-\exp ^{-\eta}}{\eta}},
$$

$\eta$ being the transverse density parameter,

$$
\eta=\frac{r_{s}^{2} N_{s}}{R^{2}}
$$

where $r_{s}$ is the string transverse radius (phenomenological estimation gives $r_{s} \sim 0.2-$ $0.3 \mathrm{fm}[10]), R^{2}$ the square of nuclear overlapping which for central collisions is equal to nuclear radius squared and $N_{s}$ is the number of produced strings. At $\eta \rightarrow 0, F(\eta) \rightarrow 1$ (no percolation/inelastic screening) and at $\eta \rightarrow \infty, F(\eta) \rightarrow 1 / \sqrt{\eta}$ (maximal screening). The detailed discussion of these behaviours can be found in [9].

In the present paper we will give an estimate for the percolation parameter $\eta$ from experimental data, not all of them connected to heavy ion physics.

Let us, in fact, start from $\gamma p$ collisions at $W \sim 200 \mathrm{GeV}$ (HERA). In lab. frame the asymmetry between comparatively slow $p$ and $\bar{p}$ was observed to be [11]

$$
A_{B}=2 \frac{N_{p}-N_{\bar{p}}}{N_{p}+N_{\bar{p}}}=(8.0 \pm 1.0 \pm 2.5) \% .
$$

That corresponds to the yield ratio

$$
R^{\gamma p}=N_{\bar{p}} / N_{p}=0.92 \pm 0.03 .
$$

However, the HERA kinematics is an asymmetrical one and particles rather slow in lab. HERA frame are rather fast in c.m. frame. To account for this we can use the QuarkGluon String Model (QGSM) [12, 13] with string junction diffusion, see details in [14]. This correction is not numerically large, the model estimation for c.m. photon-proton frame gives $R^{\gamma p}=0.86 \pm 0.02$.

In $\gamma p$ collisions we have the baryon number flux from one proton. In the case of $p p$ interactions this flux should be two times larger, that corresponding to

$$
R^{p p}=0.72-0.76
$$


in c.m. $p p$ frame.

The ratio $R^{A u A u}$ in central region of $A u A u$ collisions was measured at energy $200 \mathrm{GeV}$ per nucleon at RHIC. The values are $0.74 \pm 0.02 \pm 0.03$ [16] and $0.75 \pm 0.04$ [17], i.e. practically the same as we obtain for $p p$ collisions. This is in principle unexpected as the sea contribution, which is $\bar{p} p$ symmetrical, is much more important in heavy ion collisions. The string fusion/screening argument gives a fair explanation of what happens.

Now let us note that in the QGSM as well as in the Dual String Model (DSM) $[4,15]$ the multiplicity of every secondary $h$ produced in $p p$ collision can be written as

$$
<n_{h}^{p p}>=V_{h}+S_{h}
$$

where $V_{h}$ and $S_{h}$ are the contributions of valence and sea quarks, respectively.

In the case of heavy ion collisions the correspondent equation can be written as [18]

$$
<n_{h}^{A A}>=N_{\text {pair }} V_{h}+\left(<\nu>_{A A}-N_{\text {pair }}\right) F(\eta) S_{h},
$$

where $N_{\text {pair }}$ is the number of pair of nucleon-nucleon interactions and $\langle\nu\rangle_{A A}$ is the total number of binary interactions. $F(\eta)$ accounts for the sea strings percolation effect, a negligible effect for $p p$ collisions.

The structure of this Eq. is rather evident, for equal nuclei we have $N_{\text {pair }}$ interactions of valence quarks and diquarks (valence-valence strings) and all another $\langle\nu\rangle_{A A}-N_{\text {pair }}$ are sea quark interactions (sea-sea strings) by definitions. Of course it is true only in average, in every separate event the situation can be more complicated because the number of interacting nucleons in every nucleus can be different.

We account the percolation factor in Eq.(7) only for sea string contribution due to three reasons. First of all, the number of sea strings is in our case about 5 times larger than the number of valence strings, so percolation effects for sea strings are more important. Second and more physically important reason is that valence-valence strings (contribution $V_{h}$ in Eq. (7)) are long in rapidity space whereas sea-sea strings are rather short and their ends are distributed more close to the central region. The suppression for the inelastic screening effects coming from nuclei form factors (see [3] for details) allows the total fusion of sea-sea strings but valence-valence strings can be fused only in part, so the fusion/percolation effects will be important for the last ones only at very high energies. Third, more model dependent reason is that in DTU approach the interacting of valence quarks is considered as a first step, and after several sea quark interactions can be added, if necessary. So, the screening effects should decrease firstly the number of sea-sea strings.

In order to have $R^{p p} / R^{A A} \approx 1$ at RHIC energies, as experimentally observed, the ratio between valence quark and sea quark contributions in Eqs. (6) and (7) should be approximately the same. So we have

$$
\frac{\left(<\nu>_{A A}-N_{\text {pair }}\right) F(\eta)}{N_{\text {pair }}} \simeq 1 .
$$


Using the experimental estimates of $\langle\nu\rangle_{A A}$ and $N_{\text {pair }}$ from [19] for high- $p_{T}$ hadron production (these values are in agreement with standard Glauber-like estimates [20]) we obtain for the most central collisions the value $F(\eta)=1 / 4.8$, giving

$$
\eta=23
$$

Due to experimental errors and some model calculations we estimate the error bar in the last value to be a factor of the order 1.5. This result agrees with an estimate of $\eta$, making use of $p p$ central charged particle densities at $\sqrt{s} \simeq 200 \mathrm{GeV}, \eta \simeq 16$ (see [15]). The value obtained for $\eta$ shows that we are well inside the percolation (perhaps quark-gluon plasma) region. Note that if $R^{p p}>R^{A A}$, as it would have happened without the centre of mass correction bringing $R^{\gamma p}$ down, from $=0.92$ to 0.86 , then the $\eta$ value should have been even larger.

The contributions of valence and sea strings in QGSM for $p p$ collisions are of the same order. For $A u A u$ central interaction the sea contribution is enhanced by the factor

$$
\frac{\left\langle\nu>_{A A}-N_{\text {pair }}\right.}{N_{\text {pair }}},
$$

which is, without percolations, about 4.8. That should increase the inclusive density of secondaries. The suppression factor $F(\eta)$ about $1 / 5$ for sea contribution decreases the total rapidity density in central region about 2.5 or 3 times, that is in agreement with the previous calculations of refs. [3, 5, 21].

In our approach, the reason why the ratio $\bar{p} p$ is not much larger in heavy ion collisions is caused by the same mechanism that limits particle density in central rapidity: string fusion. It would be interesting to see if saturation models [22, 23] can also obtain a similar effect in $\bar{p} p$ suppression.

We are grateful to N.Armesto for discussions.

\section{References}

[1] V.N.Gribov. Sov.Phys.JETP 29 (1969) 483; 30 (1969) 709.

[2] O.V.Kancheli and S.G.Matinyan. Yad.Fiz. 11 (1970) 1305.

[3] A.Capella, A.Kaidalov and J.Tran Thanh Van. Heavy Ion Physics 9 (1999) 169.

[4] J.Dias de Deus and R.Ugoccioni. Phys.Lett. B494 (2000) 53.

[5] N.Armesto, C.Pajares and D.Sousa. hep-ph/0104269.

[6] N.S.Amelin, M.A.Braun and C.Pajares. Phys.Lett. B306 (1993) 312; Z.Phys. C63 (1994) 507. 
[7] M.A.Braun and C.Pajares. Phys.Rev.Lett. 85 (2000) 4864.

[8] N.Armesto, E.G.Ferreiro, C.Pajares and Yu.M.Shabelski. Z.Phys. C73 (1997) 309.

[9] M.A.Braun and C.Pajares. Eur.Phys.J. C16 (2000) 2019.

[10] N.Armesto, M.A.Braun, E.G.Ferreiro and C.Pajares. Phys.Rev.Lett. 77 (1996) 3736.

[11] C.Adloff et al. H1 Coll. Submitted to the 29th Int. Conf. on High Energy Physics ICHEP98, Vancouver, Julay 1998.

[12] A.B.Kaidalov and K.A.Ter-Martirosyan. Yad.Fiz. 39 (1984) 1545; 40 (1984) 211.

[13] A.B.Kaidalov, Yu.M.Shabelski and K.A.Ter-Martirosyan. Yad.Fiz. 43 (1986) 1282.

[14] G.H.Arakelyan, A.Capella, A.Kaidalov and Yu.M.Shabelski. hep-ph/0103337.

[15] J.Dias de Deus and R.Ugoccioni. Phys.Lett. B491 (2000) 253.

[16] PHOBOS Coll. nucl-ex/0206012.

[17] BRAMS Coll. nucl-ex/0207006.

[18] A.Armesto and C.Pajares. Int.J.Mod.Phys. A15 (2000) 2019.

[19] PHENIX Coll. nucl-ex/0207009.

[20] J.Dias de Deus and Yu.M.Shabelski. hep-ph/0107136.

[21] J.Dias de Deus, Yu.M.Shabelski and R.Ugoccioni. hep-ph/0108253.

[22] L. McLerran and R.Venugopalan. Phys.Rev. D49 (1994) 2233.

[23] A.H.Mueller and J.W.Qiu. Nucl.Phys. B268 (1986) 427. 